\title{
基于原位聚合凝胶电解质的碳纳米笼//三氧化铇纳米棒超级电容器
}

\author{
高润洲 李国昌 陈轶群 曾誉 赵杰 吴强* 杨立军王喜章 胡征 \\ (南京大学化学化工学院 介观化学教育部重点实验室 南京 210023)
}

\begin{abstract}
摘要 发展非对称超级电容器可有效提升超级电容器能量密度, 选择电极材料和电解质是关键. 分级结构碳纳米笼因 具有比表面积大、微孔-介孔-大孔共存、导电性好、稳定性高等优点, 特别适合用作超级电容器电极材料. 进一步通过 $\mathrm{N}, \mathrm{S}$ 共掺杂引入赝电容、改善浸润性，所得的氮硫共掺杂碳纳米笼 $(\mathrm{NSCNC})$ 在 $1 \mathrm{~mol} \cdot \mathrm{L}^{-1} \mathrm{H}_{2} \mathrm{SO}_{4}$ 溶液、电势范围 $0 \sim 1 \mathrm{~V}$ 、 电流密度 $1 \mathrm{~A} \cdot \mathrm{g}^{-1}$ 下表现出 $337 \mathrm{~F} \cdot \mathrm{g}^{-1}$ 的高比容量. 水合三氧化铇 $\left(\mathrm{WO}_{3} \cdot 0.6 \mathrm{H}_{2} \mathrm{O}\right)$ 纳米棒通过 $\mathrm{W}^{6^{+}} / \mathrm{W}^{5^{+}}$的氧化还原反应实 现 $\mathrm{H}^{+}$的嵌入与脱出, 在 $-0.55 \sim 0.3 \mathrm{~V} 、 5 \mathrm{~A} \cdot \mathrm{g}^{-1}$ 下表现出 $454 \mathrm{~F} \bullet \mathrm{g}^{-1}$ 的高比容量. 以 $\mathrm{NSCNC}_{\text {和 }} \mathrm{WO}_{3} \cdot 0.6 \mathrm{H}_{2} \mathrm{O}$ 作正负极材 料、原位聚合高分子凝胶电解质 $\left(\mathrm{IPGE} / \mathrm{H}_{2} \mathrm{SO}_{4}\right)$ 作准固态电解质组装的非对称超级电容器的工作电压为 $1.5 \mathrm{~V}$, 其倍率性 能非常接近于在 $\mathrm{H}$ 型电解池中以 $1 \mathrm{~mol} \cdot \mathrm{L}^{-1} \mathrm{H}_{2} \mathrm{SO}_{4}$ 为电解液的器件, 而远优于以传统聚乙烯醇/硫酸 $\left(\mathrm{PVA} / \mathrm{H}_{2} \mathrm{SO}_{4}\right)$ 作凝胶 电解质的器件, 其根源是原位聚合的 $\mathrm{IPGE} / \mathrm{H}_{2} \mathrm{SO}_{4}$ 与电极材料之间建立了有效的电荷传输界面, 改善了 $\mathrm{H}^{+}$离子的传导, 有效降低了电压降. 本工作不仅展示了酸性介质中 $\mathrm{NSCNC} / / \mathrm{WO}_{3} \cdot 0.6 \mathrm{H}_{2} \mathrm{O}$ 超级电容器的优异储能性能, 还提供了一种 新的用于构建准固态超级电容器的原位聚合凝胶电解质.
\end{abstract}

关键词 超级电容器; 酸性电解质; 原位聚合凝胶电解质; 分级结构碳纳米笼; 水合三氧化铇纳米棒

\section{Carbon Nanocages//Tungsten Trioxide Nanorods Supercapacitors with in situ Polymerized Gel Electrolytes}

\author{
Runzhou Gao Guochang Li Yiqun Chen Yu Zeng Jie Zhao Qiang Wu* \\ Lijun Yang Xizhang Wang Zheng Hu
}

(Key Laboratory of Mesoscopic Chemistry of MOE, School of Chemistry and Chemical Engineering, Nanjing University, Nanjing 210023, China)

\begin{abstract}
Asymmetric supercapacitors can effectively increase the energy density of supercapacitors, and the key is to develop high-performance electrode materials and electrolytes. Hierarchical carbon nanocages are promising electrode materials for supercapacitors because of the large specific surface area, coexisting micro-meso-macropore, good conductivity and high stability. By introducing pseudocapacitance and improving wettability via N\&S dual-doping, the N\&S dual-doped carbon nancages (NSCNC) exhibit a high specific capacity of $337 \mathrm{~F}^{\circ} \mathrm{g}^{-1}$ at $1 \mathrm{~A} \cdot \mathrm{g}^{-1}$ within the potential window of $0 \sim 1 \mathrm{~V}$ in 1 $\mathrm{mol} \cdot \mathrm{L}^{-1} \mathrm{H}_{2} \mathrm{SO}_{4}$ solution. Hydrated tungsten trioxide $\left(\mathrm{WO}_{3} \bullet 0.6 \mathrm{H}_{2} \mathrm{O}\right)$ nanorods can achieve the insertion/de-insertion of $\mathrm{H}^{+}$ via the redox reaction between $\mathrm{W}^{6^{+}} / \mathrm{W}^{5^{+}}$, and exhibit a high specific capacity of $454 \mathrm{~F} \cdot \mathrm{g}^{-1}$ at $5 \mathrm{~A} \cdot \mathrm{g}^{-1}$ within the potential window of $-0.55 \sim 0.3 \mathrm{~V}$. The solid-state asymmetric supercapacitor (SASC) with $\mathrm{NSCNC}$ and $\mathrm{WO}_{3} \bullet 0.6 \mathrm{H}_{2} \mathrm{O}$ nanorods as positive and negative electrodes, and in situ polymerized gel electrolyte (IPGE/ $\left.\mathrm{H}_{2} \mathrm{SO}_{4}\right)$ as solid electrolyte has the working voltage of $1.5 \mathrm{~V}$. And the rate performance of the SASC is very close to that of the H-type device with $1 \mathrm{~mol} \cdot \mathrm{L}^{-1} \mathrm{H}_{2} \mathrm{SO}_{4} \mathrm{So}-$ lution as electrolyte, much better than the counterpart with the traditional gel electrolyte of polyvinyl alcohol/sulfuric acid $\left(\mathrm{PVA} / \mathrm{H}_{2} \mathrm{SO}_{4}\right.$ ). The much improved SASC performance is attributed to the establishment of the effective charge transfer interface between the IPGE/ $\mathrm{H}_{2} \mathrm{SO}_{4}$ and the electrode materials, which enhances the transfer of $\mathrm{H}^{+}$ions and thereby much reduces the $I R$ drop. The energy density of the SASC with IPGE $/ \mathrm{H}_{2} \mathrm{SO}_{4}$ is $22.31 \mathrm{Wh} \cdot \mathrm{kg}^{-1}$ at $0.375 \mathrm{~kW} \cdot \mathrm{kg}^{-1}\left(0.5 \mathrm{~A} \cdot \mathrm{g}^{-1}\right)$ and $8.55 \mathrm{Wh} \cdot \mathrm{kg}^{-1}$ at $7.5 \mathrm{~kW} \cdot \mathrm{kg}^{-1}\left(10 \mathrm{~A} \cdot \mathrm{g}^{-1}\right)$, locating at the top-ranking of literatures. The SASC also exbibits excellent cycling stability, with the capacity retention of $95.2 \%$ after 4000 cycles at $5 \mathrm{~A} \cdot \mathrm{g}^{-1}$. This study not only demonstrates the excellent energy storage performance of $\mathrm{NSCNC} / / \mathrm{WO}_{3} \cdot 0.6 \mathrm{H}_{2} \mathrm{O}$ supercapacitors in acidic electrolytes, but also provides a new in-situ polymerized gel electrolyte for building high-performance SASCs.

Keywords supercapacitor; acidic electrolyte; in situ polymerized gel electrolyte; hierarchical carbon nanocage; hydrated tungsten trioxide nanorod
\end{abstract}

\footnotetext{
*E-mail:wqchem@nju.edu.cn

Received March 11, 2021; published May 17, 2021.
}

Supporting information for this article is available free of charge via the Internet at http://sioc-journal.cn.

Project supported by the National Natural Science Foundation of China (Nos. 21972061, 21832003, 21773111), the Fundamental Research Funds for the Central Universities (No. 14380237) and Nanjing University Innovation Program for PhD candidate (CXYJ21-38).

项目受国家自然科学基金(Nos. 21972061, 21832003, 21773111)、中央高校基本科研业务费专项资金(No. 14380237)和南京大学博士研究生创新研究项 目(CXYJ21-38)资助. 


\section{1 引言}

超级电容器具有功率密度高、循环寿命长等优点, 已在电子设备、交通运输等诸多领域得到广泛应用. 随 着电子器件的发展, 人们对超级电容器的能量密度、重 量、安全性和柔性等性能和参量提出了更高的要求 ${ }^{[1-2]}$, 研究兴趣逐渐着重于开发非对称、(准)固态、微型、柔 性等新型超级电容器件 ${ }^{[3-5]}$.

基于能量密度公式 $E=1 / 2 C V^{2}$, 人们通过提高电极 材料的比电容 $C$ 、拓宽工作电压 $V$ 来提升超级电容器的 能量密度 ${ }^{[6]}$. 其中, 由比电容匹配、电化学窗口互补的电 极材料组装成的非对称超级电容器可以充分发挥两种 材料的储能能力, 拓宽器件工作电压, 是获得高能量密 度的有效策略 ${ }^{[7]}$. 近年来, 本课题组发展了具有比表面 积大、微孔-介孔-大孔共存、导电性好、易于掺杂等特 征的介观结构碳纳米笼新材料, 已在能源存储与转化领 域表现出独特的优势 ${ }^{[8-11]}$, 其在水基碱性电解液和离子 液体中均表现出高比电容及优异的倍率与循环性 能 ${ }^{[1-12]}$. 研究表明, 将杂原子掺杂到碳纳米笼中可以引 入极性键改善其浸润性, 提升有效活性表面积, 还可以 产生赝电容 ${ }^{[13]}$. 然而, 掺杂碳纳米笼在酸性电解液中的 电容性能仍亟待研究 ${ }^{[14]}$. 考虑到吡啶氮、氧化噻吩硫 $\left(\mathrm{C}-\mathrm{SO}_{x}-\mathrm{C}\right)$ 等物种与氢离子的强相互作用, 氮硫共掺杂 碳纳米笼(N,S dual-doped carbon nanocages, NSCNC)可 望在酸性电解液、 $0 \sim 1 \mathrm{~V}$ 电化学窗口中表现出双电层电 容与噟电容兼容特性, 获得优异的储能性能. 为了与 $\mathrm{NSCNC}$ 的比电容和电化学窗口相匹配, 选择具有十二 角形隧道结构的 $\mathrm{WO}_{3} \cdot 0.6 \mathrm{H}_{2} \mathrm{O}$ 纳米棒作为负极材料. 该 材料可通过 $\mathrm{W}^{6+} / \mathrm{W}^{5+}$ 之间的氧化还原反应实现质子/水 合质子在隧道中的快速嵌入与脱出 ${ }^{[15]}$, 在 $-0.55 \sim 0.3 \mathrm{~V}$ 电化学窗口中的理论比电容可达 $466 \mathrm{~F} \cdot \mathrm{g}^{-1}\left(396 \mathrm{C} \cdot \mathrm{g}^{-1}\right)$. 以这两种电极材料构建非对称超级电容器, 可望获得约 $1.5 \mathrm{~V}$ 的工作电压和高比电容.

另一方面, 采用准固态电解质(例如聚合物凝胶电 解质等) 代替液态电解质, 可以防止有害液态电解质的 泄露, 提高安全性, 也为电子设备的设计带来根本性的 变化, 尤其在柔性、微型化和适应性方面优势明显 ${ }^{[16-18]}$. 聚合物凝胶电解质的常用基体有聚乙烯醇(PVA)、聚甲 基丙烯酸甲酯、聚偏氟乙烯、聚丙烯腈等 ${ }^{[17]}$. 通常情况 下, 聚合物与液态电解质先形成凝胶, 再涂覆到固体电 极材料表面. 这会导致电极材料与聚合物凝胶电解质之 间难以建立有效的电荷传输界面, 甚至部分电极材料不 能与凝胶电解质接触, 从而增加器件的等效串联电阻, 降低活性材料的利用率 ${ }^{[19]}$. 以这种方式构建的聚合物 凝胶电解质器件与相应的液态电解质器件相比性能通 常有显著差异 ${ }^{[20]}$. 如果先配制聚合物的单体与导电介 质(如 $\mathrm{H}_{2} \mathrm{SO}_{4}$ ) 的溶液, 使之均匀渗透到电极材料层中, 再原位聚合形成凝胶电解质, 则可使所有电极材料的表 面与凝胶电解质充分接触, 建立有效的电荷传输界面,
从而获得与液态电解质体系相近的储能性能. 该思路已 应用于锂/钠离子电池、超级电容器等器件, 在一定程度 上改善了(准)固态储能器件的性能 ${ }^{[16,21]}$.

基于以上考虑, 本工作制得了 $\mathrm{NSCNC}$ 和 $\mathrm{WO}_{3}$ ・ $0.6 \mathrm{H}_{2} \mathrm{O}$ 纳米棒, 采用三电极系统在 $1.0 \mathrm{~mol} \cdot \mathrm{L}^{-1} \mathrm{H}_{2} \mathrm{SO}_{4}$ 溶 液中分别测试其电容性能, 并在 $\mathrm{H}$ 型电解池中采用两电 极系统测试了 $\mathrm{NSCNC} / / \mathrm{WO}_{3} \bullet 0.6 \mathrm{H}_{2} \mathrm{O}$ 非对称超级电容器 的性能. 进而通过丙烯酰胺和 $N, N^{\prime}$-亚甲基双丙烯酰胺 在 $1.0 \mathrm{~mol} \cdot \mathrm{L}^{-1} \mathrm{H}_{2} \mathrm{SO}_{4}$ 溶液中的原位共聚反应构建聚合 物凝胶电解质 (IPGE $\left./ \mathrm{H}_{2} \mathrm{SO}_{4}\right)$, 组装了 $\mathrm{NSCNC} / / \mathrm{WO}_{3}$ $0.6 \mathrm{H}_{2} \mathrm{O}$ 准固态超级电容器, 性能可与液态电解质超级 电容器的性能相媲美, 其倍率和循环性能远优于以传统 $\mathrm{PVA} / \mathrm{H}_{2} \mathrm{SO}_{4}$ 凝胶电解质组装的 $\mathrm{NSCNC} / / \mathrm{WO}_{3} \cdot 0.6 \mathrm{H}_{2} \mathrm{O}$ 器 件. 本研究不仅展示了酸性介质中 $\mathrm{NSCNC} / / \mathrm{WO}_{3}$ ・ $0.6 \mathrm{H}_{2} \mathrm{O}$ 超级电容器的优异储能性能, 还提供了一种新 的用于构建准固态超级电容器的原位聚合凝胶电解质.

\section{2 结果与讨论}

图 1 为 $\mathrm{NSCNC}$ 和 $\mathrm{WO}_{3} \cdot 0.6 \mathrm{H}_{2} \mathrm{O}$ 的形貌与结构表征 结果. 扫描电镜 (SEM) 和透射电镜 (TEM) 照片显示, NSCNC 是由 5 25 nm 的空心纳米笼相连成纳米片、进 而交叠成介观分级结构(图 1a), 与我们之前报道的碳纳 米笼的形貌一致 ${ }^{[12]} . \mathrm{X}$ 射线光电子能谱(XPS)表征显示 NSCNC 中含有 C (81.45 at \%)、N (9.91 at \%)、S (2.27 at \%) 元素. S $2 p$ 精细谱可拟合为两组分裂峰(图 1b), 结合能 位于 163.8 和 $164.9 \mathrm{eV}$ 的 $2 \mathrm{p}_{3 / 2}$ 和 $2 \mathrm{p}_{1 / 2}$ 分裂峰可归属为 噻吩硫(C-S-C), 位于 167.8 和 $169.0 \mathrm{eV}$ 的分裂峰可归属 为氧化噻吩硫 $\left(\mathrm{C}-\mathrm{SO}_{x}-\mathrm{C}\right)^{[22]} . \mathrm{N} 1 \mathrm{~s}$ 精细谱可拟合为位于 398.4、400.0、401.1 和 $403.5 \mathrm{eV}$ 的 N1、N2、N3、N4 峰(图 1c), 依次对应于吡啶氮、吡咯氮、石墨氮和氧化 吡啶氮 ${ }^{[13]}$. NSCNC 的 X 射线衍射 (XRD)谱显示含有 (002)衍射峰, 表明其具有较好的石墨化程度(图 S1), 四 探针测试显示其体相电导率为 $156.3 \mathrm{~S} \cdot \mathrm{m}^{-1}$. $\mathrm{N}_{2}$ 吸附-脱 附测试表明 NSCNC 的比表面积可达 $2298 \mathrm{~m}^{2} \bullet \mathrm{g}^{-1}$, 含有 丰富的微孔、介孔和大孔(图 S2). NSCNC 的高比表面积 有利于电荷存储, 高导电性和多级孔结构有利于电荷传 输, 而 $\mathrm{N} 、 \mathrm{~S}$ 共掺杂可改善其表面浸润性(图 S3) ${ }^{[12]}$, 这 些结构特征可赋予 NSCNC 优良的电容性能.

$\mathrm{TEM}$ 表征显示 $\mathrm{WO}_{3} \cdot 0.6 \mathrm{H}_{2} \mathrm{O}$ 具有纳米棒状形貌, 直 径约为 $10 \sim 20 \mathrm{~nm}$, 长度可达微米级(图 1d). 高分辨 TEM 照片显示其具有较好的结晶度, 相邻晶格条纹之 间的间距为 $0.379 \mathrm{~nm}$, 对应于 $\mathrm{WO}_{3} \cdot 0.6 \mathrm{H}_{2} \mathrm{O}$ 的 $(001)$ 晶面, 表明纳米棒沿着 [001]方向生长. XRD 衍射峰与 $\mathrm{WO}_{3} \cdot 0.6 \mathrm{H}_{2} \mathrm{O}$ 的标准谱图(PDF\# 75-2187)相吻合(图 1e). 热重分析结果表明每个结构单元的晶格水含量约为 0.6 个, 进一步表明其组成为 $\mathrm{WO}_{3} \cdot 0.6 \mathrm{H}_{2} \mathrm{O}$ (图 S4). XPS W4f 谱中位于 36.15 和 $38.3 \mathrm{eV}$ 的信号分别对应于 $\mathrm{W}^{6+}$ 的 $4 \mathrm{f}_{7 / 2}$ 和 $4 \mathrm{f}_{5 / 2}{ }^{[15]}$, 表明样品中 $\mathrm{W}$ 物种以 +6 形式存在, 跟 $\mathrm{WO}_{3}$ 
组成相吻合(图 1f). $\mathrm{WO}_{3} \cdot 0.6 \mathrm{H}_{2} \mathrm{O}$ 的 $\mathrm{N}_{2}$ 吸附-脱附测试结 果显示其比表面积为 $183.5 \mathrm{~m}^{2} \cdot \mathrm{g}^{-1}$ (图 S5).

采用三电极系统在 $1.0 \mathrm{~mol} \cdot \mathrm{L}^{-1} \mathrm{H}_{2} \mathrm{SO}_{4}$ 溶液中分别 测试了 $\mathrm{NSCNC}$ 和 $\mathrm{WO}_{3} \cdot 0.6 \mathrm{H}_{2} \mathrm{O}$ 的电化学储能性能, 结 果分别示于图 2 和图 3 中. NSCNC 的循环伏安曲线 $(\mathrm{CV})$ 在 $0 \sim 1 \mathrm{~V}$ (vs. $\mathrm{Ag} / \mathrm{AgCl}$ ) 电化学窗口内近似为矩形, 在约 $0.4 \mathrm{~V}$ 附近有一对峰电位相差小的氧化还原峰, 表明 $\mathrm{NSCNC}$ 的电荷存储以双电层电容行为为主, 同时存在 一定的赝电容贡献(图 2a 和图 S6) ${ }^{[23]}$. 氧化还原峰来自 于表面吡啶氮等物种的质子化与去质子化, 其强度随扫 速增加逐渐变弱. NSCNC 在不同电流密度下的恒电流 充放电曲线(GCD)基本呈线性变化(图 2b), 进一步表明 其双电层电容为主的储能行为. 根据公式 $C_{\mathrm{wt}}=$ $(I \Delta t) /[m(\Delta V-I R)]$, 可计算得到不同电流密度下的质量
比电容(其中, $I R$ 是放电曲线的电压降), 示于图 $2 \mathrm{c}$. 在电 流密度 $1 \mathrm{~A} \cdot \mathrm{g}^{-1}$ 下, NSCNC 的比电容达 $337 \mathrm{~F} \cdot \mathrm{g}^{-1}$, 在 50

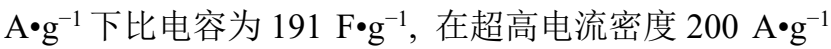
仍可保留 $68 \mathrm{~F} \bullet \mathrm{g}^{-1}$, 表明 NSCNC 具有高比电容和优良的 倍率性能. 由 NSCNC 的交流阻抗谱(EIS)可知(图 S7), 接触阻抗 $R_{\mathrm{s}}$ 为 $1.14 \Omega$, 电荷转移阻抗 $R_{\mathrm{ct}}$ 为 $1.33 \Omega$, Warburg 阻抗 $R_{\mathrm{W}}$ 为 $0.14 \Omega$, 等效串联电阻 $R_{\mathrm{ESR}}$ 为 $2.61 \Omega$. 较小的 $R_{\mathrm{ESR}}$ 表明 $\mathrm{NSCNC}$ 具有良好的电荷传输动力 学 ${ }^{[24-25]}$, 这与其高导电性和介观多孔结构非常有利于电 荷传输有关. 优良的电荷传输动力学赋予 NSCNC 优良 的倍率性能. 在 $20 \mathrm{~A} \cdot \mathrm{g}^{-1}$ 下测试了循环稳定性, 在循环 10000 圈之后, NSCNC 的比电容保持率和库伦效率均> 98\%(图 2d), 测试过程中的 EIS 谱无明显变化, 即 $R_{\mathrm{ESR}}$ 保持稳定(图 S8). 循环测试后 NSCNC 的形貌和物相未
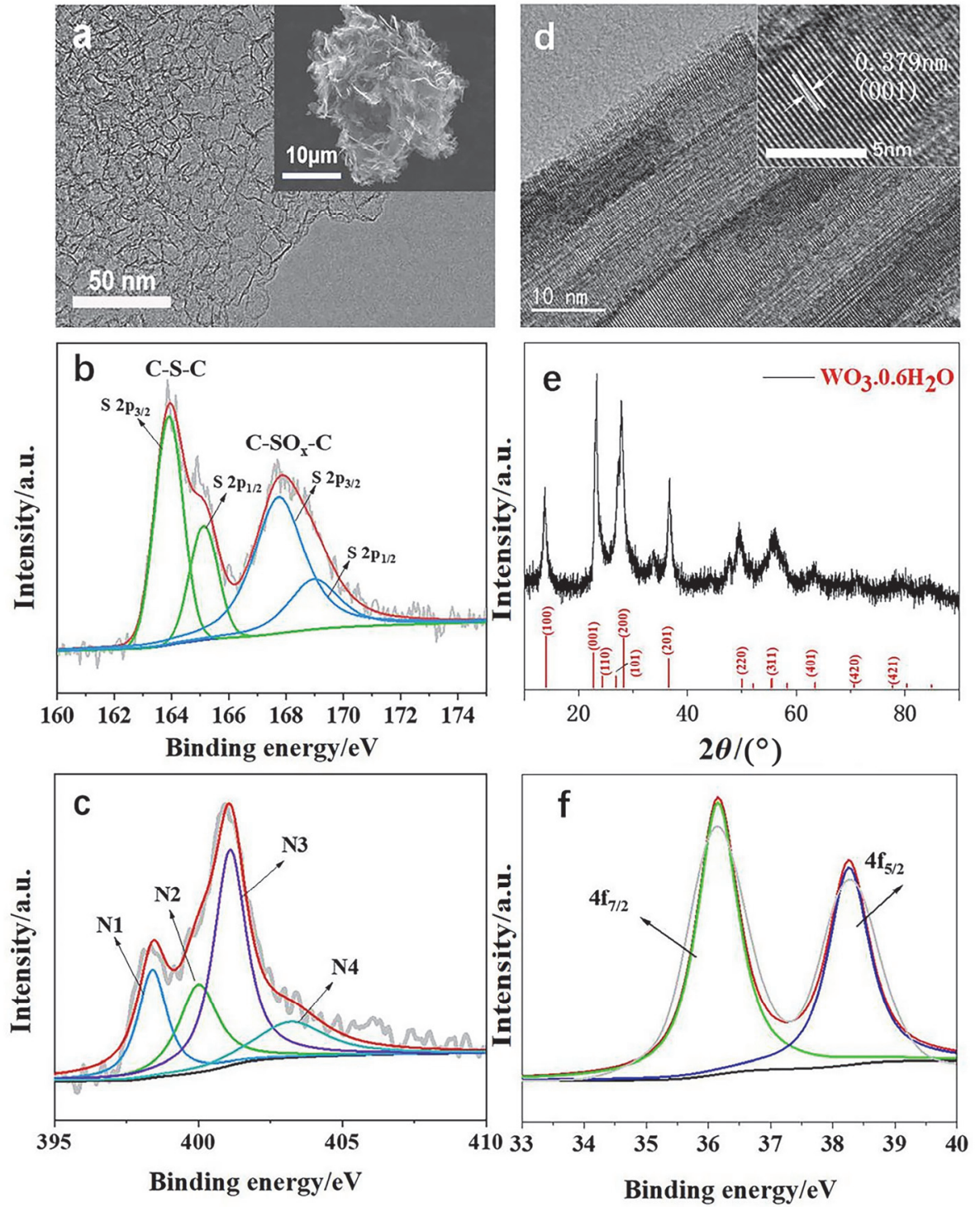

图 $1 \mathrm{NSCNC}$ 和 $\mathrm{WO}_{3} \bullet 0.6 \mathrm{H}_{2} \mathrm{O}$ 纳米棒的形貌与结构表征. (a) NSCNC 的 SEM 和 TEM 照片, (b) S2p 和(c) N1s XPS 谱图. (d) WO $\mathrm{WO}_{3} \cdot 0.6 \mathrm{H}_{2} \mathrm{O}$ 的 TEM 照片, (e) XRD 谱和(f) W4f XPS 谱图.

Figure 1 Morphology and structure characterizations of NSCNC and $\mathrm{WO}_{3} \bullet 0.6 \mathrm{H}_{2} \mathrm{O}$ nanorods. (a) SEM and TEM images, (b) S2p and (c) N1s XPS spectra of NSCNC. (d) TEM image, (e) XRD pattern and (f) W4f XPS spectrum of $\mathrm{WO}_{3} \cdot 0.6 \mathrm{H}_{2} \mathrm{O}$ nanorods. 

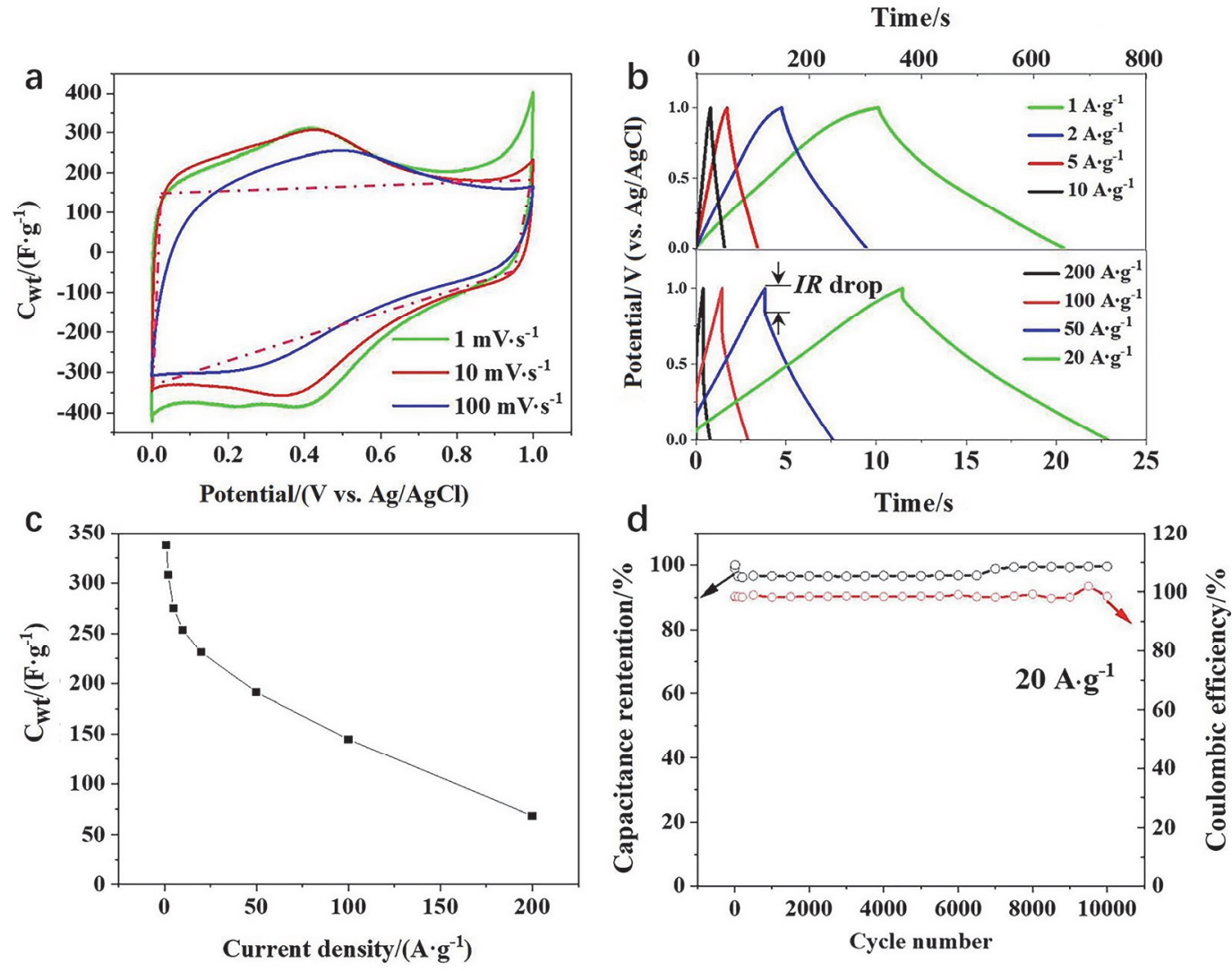

图 2 NSCNC 的电化学性能. (a) CV 曲线. (b) GCD 曲线. (c) 比电容与电流密度关系曲线. (d) 循环稳定性和库伦效率.

Figure 2 Electrochemical performance of NSCNC. (a) CV curves. (b) GCD curves. (c) Relationship of capacitance vs. current density. (d) Capacitance retention and Coulombic efficiency during cycling test.

发生明显改变(图 S9). 这些测试表明 NSCNC 具有优异 的循环稳定性.

$\mathrm{CV}$ 曲线显示 $\mathrm{WO}_{3} \cdot 0.6 \mathrm{H}_{2} \mathrm{O}$ 在 $-0.55 \sim 0.3 \mathrm{~V}$ (vs. $\mathrm{Ag} / \mathrm{AgCl}$ )电化学窗口工作, 在低扫速(例如 5、10、20 $\mathrm{mV} \cdot \mathrm{s}^{-1}$ )下具有 3 组可逆的氧化还原峰(图 3a), 其中三个 还原峰与文献报道的 $\mathrm{WO}_{3} \cdot 0.6 \mathrm{H}_{2} \mathrm{O}$ 三步质子化过程相吻 合 ${ }^{[15]}$, 即约 0.25 个晶格水从 $\mathrm{WO}_{3} \cdot 0.89 \mathrm{H}_{2} \mathrm{O}$ (由 $\mathrm{WO}_{3} \cdot 0.6 \mathrm{H}_{2} \mathrm{O}$ 自发吸附水分子形成)中排出的同时插入 0.25 个质子 $(0 \sim-0.25 \mathrm{~V})$ 、约 0.30 个裸质子嵌入隧道中 $(-0.25 \sim-0.40 \mathrm{~V})$ 、约 0.17 个 $\mathrm{H}_{3} \mathrm{O}^{+}$插入隧道 $(-0.40 \sim$ $-0.55 \mathrm{~V})$. 当 $\mathrm{CV}$ 的扫速增大到 $50 \mathrm{mV} \cdot \mathrm{s}^{-1}$ 以上, 氧化还 原峰基本消失, 此时 $\mathrm{WO}_{3} \cdot 0.6 \mathrm{H}_{2} \mathrm{O}$ 主要通过表面双电层 电容行为存储电荷. $\mathrm{WO}_{3} \cdot 0.6 \mathrm{H}_{2} \mathrm{O}$ 在不同电流密度下的 GCD 曲线存在电压快速下降和缓慢下降两个区间(图 $3 b)$, 跟 CV 曲线相对应. 随电流密度增加, 电压降显著 增加. 基于 $\mathrm{GCD}$ 放电曲线可计算得到 $\mathrm{WO}_{3} \cdot 0.6 \mathrm{H}_{2} \mathrm{O}$ 的比 电容(图 3c), 在 $5 \mathrm{~A} \cdot \mathrm{g}^{-1}$ 下比电容达 $454 \mathrm{~F} \cdot \mathrm{g}^{-1}$, 非常接近 $\mathrm{WO}_{3} \cdot 0.6 \mathrm{H}_{2} \mathrm{O}$ 的理论比电容. 在 $50 \mathrm{~A} \cdot \mathrm{g}^{-1}$ 下比电容为 266 $\mathrm{F} \cdot \mathrm{g}^{-1}$, 在超高电流密度 $200 \mathrm{~A} \cdot \mathrm{g}^{-1}$ 下仍可保留 $144 \mathrm{~F} \bullet \mathrm{g}^{-1}$ 的比电容, 可见其具有优秀的倍率性能. $20 \mathrm{~A} \cdot \mathrm{g}^{-1}$ 下循环 9000 圈之后, $\mathrm{WO}_{3} \cdot 0.6 \mathrm{H}_{2} \mathrm{O}$ 的比电容保持率为 $98 \%$, 库伦 效率 $>95 \%$, 即具有优异的循环稳定性(图 3d). 循环测
试后 $\mathrm{WO}_{3} \cdot 0.6 \mathrm{H}_{2} \mathrm{O}$ 的形貌和物相未发生明显改变(图 S10).

上述结果表明, $\mathrm{NSCNC}$ 和 $\mathrm{WO}_{3} \cdot 0.6 \mathrm{H}_{2} \mathrm{O}$ 的电化学窗 口分别为 $0 \sim 1 \mathrm{~V}$ 和 $-0.55 \sim 0.3 \mathrm{~V}$, 且具有较为匹配的 比容量和倍率性能. 本工作分别以 NSCNC 和 $\mathrm{WO}_{3} \cdot 0.6 \mathrm{H}_{2} \mathrm{O}$ 作为正负极材料, 采用 $\mathrm{IPGE} / \mathrm{H}_{2} \mathrm{SO}_{4}$ 凝胶电 解质组装了准固态超级电容器(图 $\mathrm{S} 11$ ), 并与在 $\mathrm{H}$ 型电 解池中以 $1.0 \mathrm{~mol} \cdot \mathrm{L}^{-1} \mathrm{H}_{2} \mathrm{SO}_{4}$ 溶液为电解质的超级电容 器以及以传统 $\mathrm{PVA} / \mathrm{H}_{2} \mathrm{SO}_{4}$ 凝胶为电解质的超级电容器 的性能进行对比, 揭示 $\mathrm{IPGE} / \mathrm{H}_{2} \mathrm{SO}_{4}$ 凝胶电解质在(准) 固态超级电容器性能发挥中的促进作用.

图 4 为 $\mathrm{IPGE} / \mathrm{H}_{2} \mathrm{SO}_{4}$ 凝胶的原位聚合形成及其与电 极材料的接触界面示意图. 称取适量的丙烯酰胺和 $N, N^{\prime}$-亚甲基双丙烯酰胺溶解于 $1.0 \mathrm{~mol} \cdot \mathrm{L}^{-1} \mathrm{H}_{2} \mathrm{SO}_{4}$ 中, 加 入适量双氧水和抗坏血酸溶液作为引发剂, 形成的前驱 液澄清透明、流动性好. 演示实验显示该前驱液在 $50{ }^{\circ} \mathrm{C}$ 下放置 $30 \mathrm{~min}$ 即可交联共聚形成 $\mathrm{IPGE} / \mathrm{H}_{2} \mathrm{SO}_{4}$ 水 凝胶(图 4a, 图 S12). SEM 照片显示 $\mathrm{IPGE} / \mathrm{H}_{2} \mathrm{SO}_{4}$ 水凝胶 中含有丰富的分级孔道, $\mathrm{H}_{2} \mathrm{SO}_{4}$ 水溶液通过氢键被锁定 在这些孔道中. 采用正负极电荷平衡公式 $m_{+} / m_{-}=$ $C . V_{-} / C_{+} V_{+}{ }^{[26]}$ 计算得 $\mathrm{NSCNC}$ 和 $\mathrm{WO}_{3} \cdot 0.6 \mathrm{H}_{2} \mathrm{O}$ 电极的质量 比 $m_{+} / m_{-}$为 $1.32: 1$. 将两种电极材料做成浆料, 均匀地 

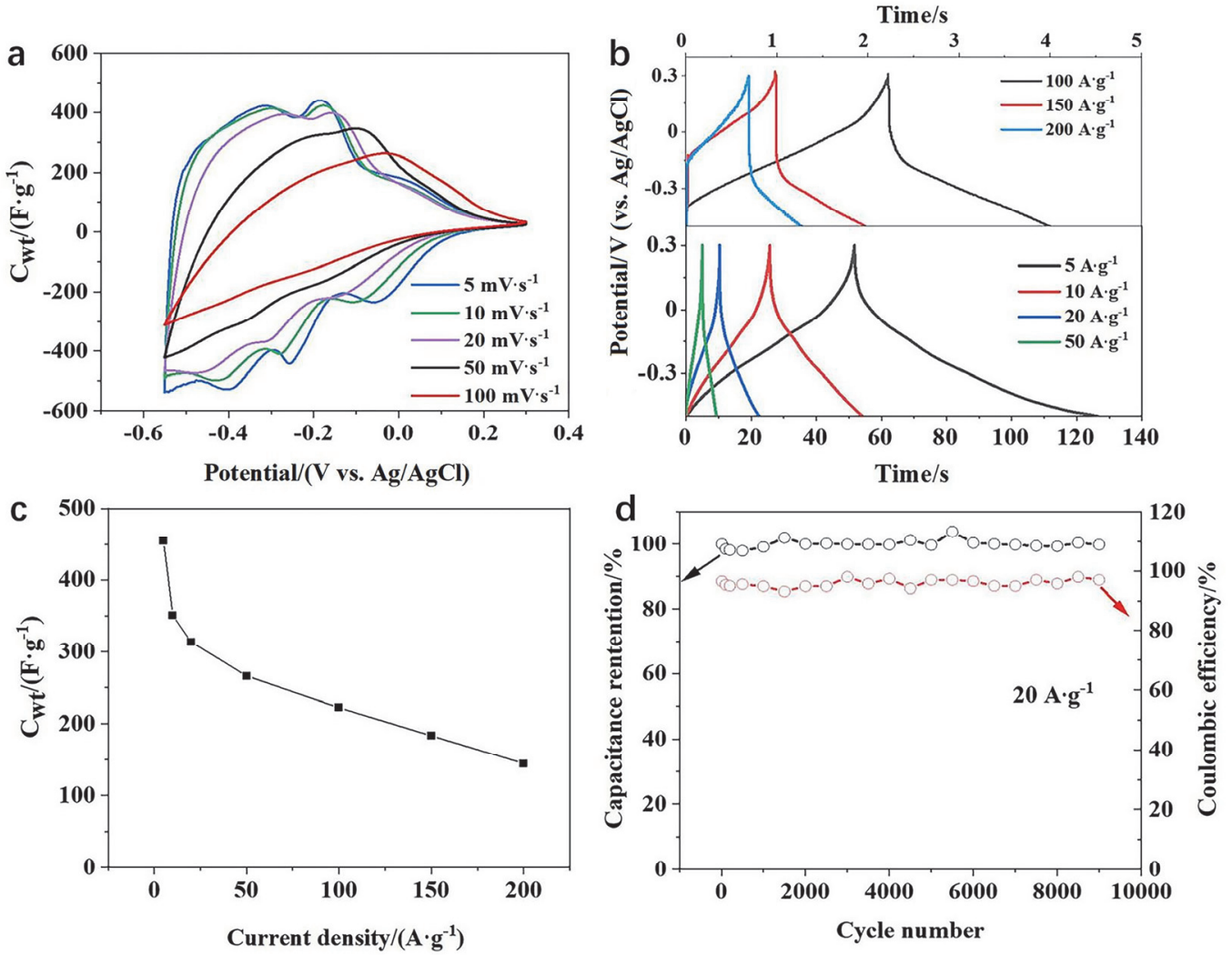

图 $3 \mathrm{WO}_{3} \bullet 0.6 \mathrm{H}_{2} \mathrm{O}$ 纳米棒的电化学性能. (a) $\mathrm{CV}$ 曲线. (b) $\mathrm{GCD}$ 曲线. (c) 比电容-电流密度关系曲线. (d) 循环稳定性和库伦效率.

Figure 3 Electrochemical performance of $\mathrm{WO}_{3} \cdot 0.6 \mathrm{H}_{2} \mathrm{O}$ nanorods. (a) $\mathrm{CV}$ curves. (b) GCD curves. (c) Relationship of capacitance vs. current density. (d) Capacitance retention and Coulombic efficiency during cycling test.

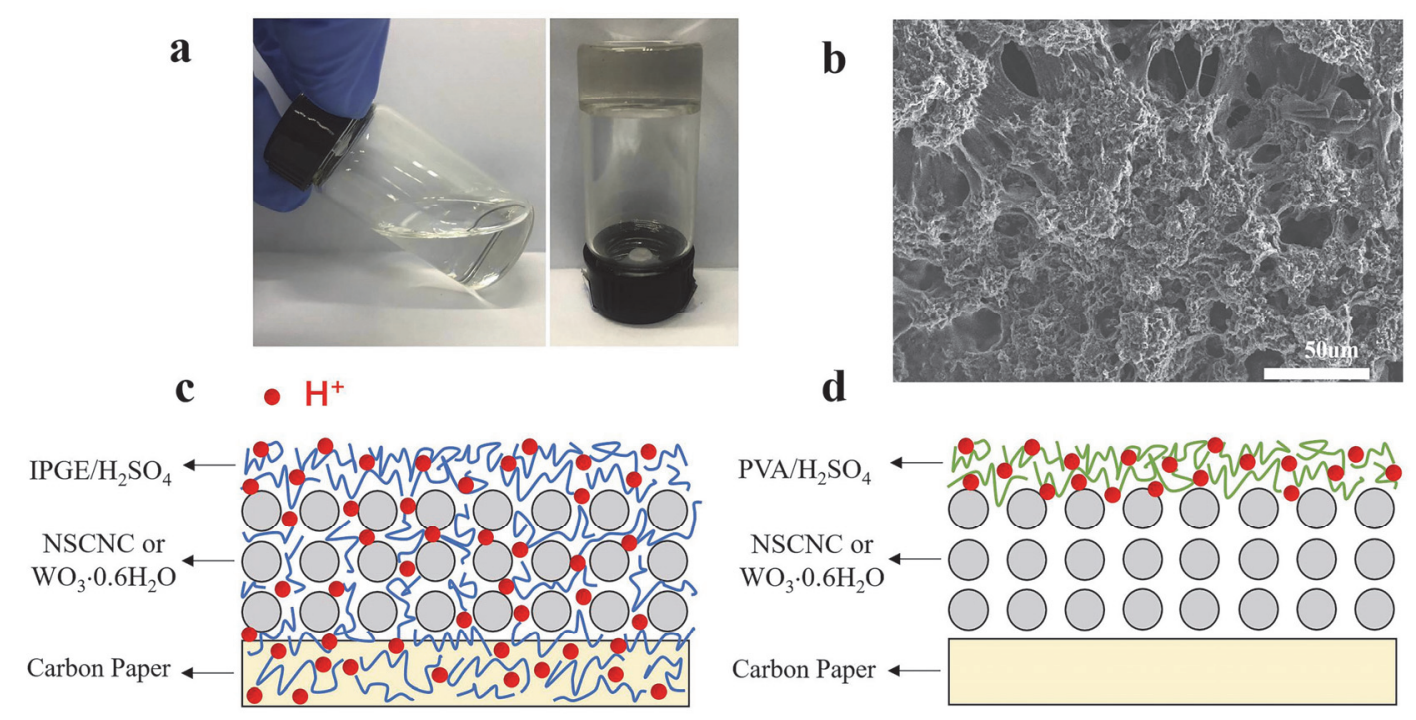

图 $4 \mathrm{IPGE} / \mathrm{H}_{2} \mathrm{SO}_{4}$ 的原位聚合形成及其与电极材料的接触界面示意图. (a) 前驱液与 IPGE/ $\mathrm{H}_{2} \mathrm{SO}_{4}$ 凝胶的照片. (b) $\mathrm{NSCNC}$ 与 $\mathrm{IPGE} / \mathrm{H}_{2} \mathrm{SO}_{4}$ 形成的电 极的 SEM 照片. (c) IPGE $/ \mathrm{H}_{2} \mathrm{SO}_{4}$ 与电极材料的界面示意图. (d) $\mathrm{PVA} / \mathrm{H}_{2} \mathrm{SO}_{4}$ 与电极材料界面示意图

Figure 4 Formation of IPGE/ $\mathrm{H}_{2} \mathrm{SO}_{4}$ and schematic diagrams of interfaces of gel electrolyte-electrode materials. (a) Photographs of the precursor solution and IPGE/ $/ \mathrm{H}_{2} \mathrm{SO}_{4}$. (b) SEM image of IPGE/ $\mathrm{H}_{2} \mathrm{SO}_{4}-\mathrm{NSCNC}$ electrode. (c) Schematic diagram of IPGE/ $/ \mathrm{H}_{2} \mathrm{SO}_{4}$-electrode materials interface. (d) Schematic diagram of $\mathrm{PVA} / \mathrm{H}_{2} \mathrm{SO}_{4}$-electrode materials interface

涂覆到碳纸表面，干燥后浸入上述前驱液中，使之充分 浸润到电极材料层内部, 加热即可原位聚合形成多孔水 凝胶(图 4b, 图 S13). 由于前驱液流动性好, 可渗透到电
极材料层内部，原位聚合后在电极材料的间隙中形成 $\mathrm{IPGE} / \mathrm{H}_{2} \mathrm{SO}_{4}$ 凝胶，可保证所有电极材料的表面与凝胶 电解质充分接触，建立有效的电荷传输界面(图 4c). 相 
对地, 传统的(准)固态 $\mathrm{PVA} / \mathrm{H}_{2} \mathrm{SO}_{4}$ 电解质是预先形成凝 胶, 其流动性和渗透性很差, 只能与表层的电极材料接 触(图 4d), 使部分电极材料没有接触到凝胶电解质而不 能发挥储能作用.

测试了液态非对称超级电容器及以 $\mathrm{IPGE} / \mathrm{H}_{2} \mathrm{SO}_{4}$ 和 $\mathrm{PVA} / \mathrm{H}_{2} \mathrm{SO}_{4}$ 为凝胶电解质的(准)固态超级电容器的电化 学性能. 根据器件的 $\mathrm{CV}$ 曲线, 其峰面积的变化趋势为: 液态电解质器件 $\approx I P G E / \mathrm{H}_{2} \mathrm{SO}_{4}$ 器件 $>\mathrm{PVA} / \mathrm{H}_{2} \mathrm{SO}_{4}$ 器件 (图 S14 S16). 各器件的倍率性能、循环性能测试结果 示于图 5. 根据 GCD 曲线可知, 三种器件的工作电压均 为 $1.5 \mathrm{~V}$ (图 $\mathrm{S} 17 \sim \mathrm{S} 19$ ). 基于正负极电极材料总质量计 算得到了不同电流密度下的质量比电容(图 5a, 图 S21). 在 $1 、 5 、 20 \mathrm{~A} \cdot \mathrm{g}^{-1}$ 时, 液态电解液器件的比电容分别为 74.4、65.3、50.6 F・g ${ }^{-1}, \mathrm{IPGE} / \mathrm{H}_{2} \mathrm{SO}_{4}$ 器件的比电容分别 为 71.3、58.9、52.6 F• g ${ }^{-1}, \mathrm{PVA} / \mathrm{H}_{2} \mathrm{SO}_{4}$ 器件的比电容分 别为 $63.9 、 32.6 、 0 \mathrm{~F} \cdot \mathrm{g}^{-1}$. 可见, $\mathrm{IPGE} / \mathrm{H}_{2} \mathrm{SO}_{4}$ 器件的比电 容非常接近于液态电解液器件, 而 $\mathrm{PVA} / \mathrm{H}_{2} \mathrm{SO}_{4}$ 器件的比 电容在高倍率下显著下降. 三种器件倍率性能的差异源 于其不同的电荷传输动力学. 从不同倍率的 GCD 曲线 可知, 液态电解液器件的电压降 (IR drop) 最小, 凝胶电 解质器件的电压降相对增加, 其中 $\mathrm{IPGE} / \mathrm{H}_{2} \mathrm{SO}_{4}$ 器件的
电压降比 $\mathrm{PVA} / \mathrm{H}_{2} \mathrm{SO}_{4}$ 器件明显要小(图 5b), 表明前者比 后者具有更优的电荷传输动力学. EIS 测试结果显示, IPGE/ $\mathrm{H}_{2} \mathrm{SO}_{4}$ 器件的 $R_{\mathrm{S}} 、 R_{\mathrm{ct}}$ 和 $R_{\mathrm{w}}$ 均小于 $\mathrm{PVA} / \mathrm{H}_{2} \mathrm{SO}_{4}$ 器 件的对应值, 尤其是 $R_{\mathrm{ct}}$ 要小得多, 表明 $\mathrm{IPGE} / \mathrm{H}_{2} \mathrm{SO}_{4}$ 与 电极材料形成了有效的电荷传输界面, 远优于 $\mathrm{PVA} / \mathrm{H}_{2} \mathrm{SO}_{4}$ 器件中部分接触的情形(图 $4 \mathrm{c}, 4 \mathrm{~d}$ ), 导致其 等效串联电阻与放电过程电压降减小(图 S20, 表 S1).

三种 $\mathrm{NSCNC} / / \mathrm{WO}_{3} \cdot 0.6 \mathrm{H}_{2} \mathrm{O}$ 非对称电容器的 Ragone 图显示(图 5c), 液态电解质器件在功率密度 0.375 $\mathrm{kW} \cdot \mathrm{kg}^{-1}\left(0.5 \mathrm{~A} \cdot \mathrm{g}^{-1}\right)$ 时能量密度达到 $24.59 \mathrm{Wh} \cdot \mathrm{kg}^{-1}$, 在 功率密度 $7.5 \mathrm{~kW} \cdot \mathrm{kg}^{-1}\left(10 \mathrm{~A} \cdot \mathrm{g}^{-1}\right)$ 时能量密度为 18.33 $\mathrm{Wh} \bullet \mathrm{kg}^{-1}$; IPGE $/ \mathrm{H}_{2} \mathrm{SO}_{4}$ 器件在功率密度 $0.375 \mathrm{~kW} \cdot \mathrm{kg}^{-1}$ $\left(0.5 \mathrm{~A} \cdot \mathrm{g}^{-1}\right)$ 时能量密度达到 $22.31 \mathrm{Wh} \cdot \mathrm{kg}^{-1}$, 在功率密度 $7.5 \mathrm{~kW} \cdot \mathrm{kg}^{-1}\left(10 \mathrm{~A} \cdot \mathrm{g}^{-1}\right)$ 时能量密度为 $8.55 \mathrm{Wh} \cdot \mathrm{kg}^{-1}$. 相对 地, $\mathrm{PVA} / \mathrm{H}_{2} \mathrm{SO}_{4}$ 器件在功率密度 $0.375 \mathrm{~kW} \cdot \mathrm{kg}^{-1}(0.5$ $\left.\mathrm{A} \cdot \mathrm{g}^{-1}\right)$ 时能量密度为 $22.77 \mathrm{Wh} \cdot \mathrm{kg}^{-1}$, 在功率密度 7.5 $\mathrm{kW} \cdot \mathrm{kg}^{-1}\left(10 \mathrm{~A} \cdot \mathrm{g}^{-1}\right)$ 时能量密度仅为 $0.18 \mathrm{Wh} \cdot \mathrm{kg}^{-1}$. 可见， $\mathrm{IPGE} / \mathrm{H}_{2} \mathrm{SO}_{4}$ 器件的高倍率性能虽然比不上液态电解液 器件, 但远优于传统的 $\mathrm{PVA} / \mathrm{H}_{2} \mathrm{SO}_{4}$ 器件. IPGE/ $\mathrm{H}_{2} \mathrm{SO}_{4}$ 器 件的能量密度也高于文献报道的 $\mathrm{NiO} / / \alpha-\mathrm{Fe}_{2} \mathrm{O}_{3}{ }^{[27]}$ 、 $\mathrm{CoS} / \mathrm{AC}^{[28]}$ 等固态器件和 $\mathrm{NaTi}_{2}\left(\mathrm{PO}_{4}\right)_{3} / \mathrm{C} / / \mathrm{AC}^{[29]}$ 等液态器
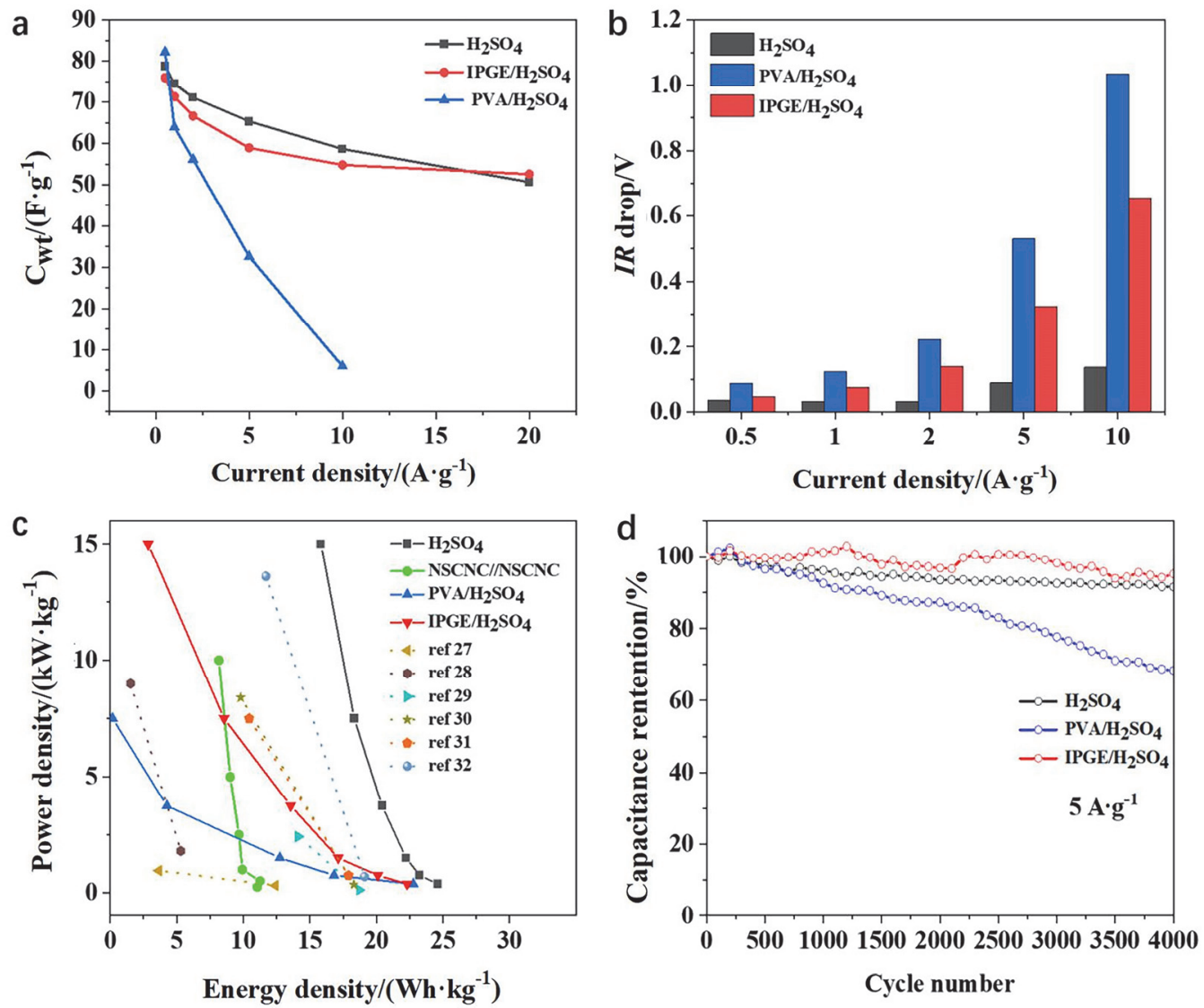

图 5 三种非对称电容器的电化学性能. (a) 比电容-电流密度关系曲线. (b) 不同电流密度下 GCD 曲线的电压降. (c) Ragone 曲线. (d) 循环稳定性. Figure 5 Electrochemical performance of three asymmetric supercapacitors. (a) Relationships of capacitance vs. current density. (b) IR drops at different current densities. (c) Ragone plots. (d) Cycling stabilities. 
件的能量密度, 可婫美于 $\mathrm{CPY} / / \mathrm{C}-\mathrm{G} / \mathrm{AFC}{ }^{[30]}$ 、 $\mathrm{Co}_{3} \mathrm{O}_{4}-\mathrm{NF} / / \mathrm{CA}^{[31]}$ 等固态器件, 但仍低于先进的 meso- $\mathrm{NiO} / \mathrm{Ni} / / \mathrm{CNCs}^{[32]}$ 等液态器件. 此外, 在 $5 \mathrm{~A} \cdot \mathrm{g}^{-1}$ 下 循环 4000 圈后, IPGE/ $\mathrm{H}_{2} \mathrm{SO}_{4}$ 器件的比电容保持率为 $95.2 \%$, 明显高于 $\mathrm{PVA} / \mathrm{H}_{2} \mathrm{SO}_{4}$ 器件的 $68.3 \%$, 甚至优于液 态电解液器件的 $91.5 \%$. 该结果表明, IPGE $/ \mathrm{H}_{2} \mathrm{SO}_{4}$ 器件 的循环稳定性远优于传统的 $\mathrm{PVA} / \mathrm{H}_{2} \mathrm{SO}_{4}$ 器件, 这源于 $\mathrm{IPGE} / \mathrm{H}_{2} \mathrm{SO}_{4}$ 凝胶的优异锁水性能及其与电极材料之间 稳定的电荷传输界面.

当正极 $\mathrm{NSCNC}$ 的负载量从 $1.0 \mathrm{mg} \cdot \mathrm{cm}^{-2}$ 增加到 5.0 $\mathrm{mg} \cdot \mathrm{cm}^{-2}$ 时 (负极 $\mathrm{WO}_{3} \cdot 0.6 \mathrm{H}_{2} \mathrm{O}$ 的负载量相应增加), $\mathrm{IPGE} / \mathrm{H}_{2} \mathrm{SO}_{4}$ 器件的比电容、能量/功率密度和循环稳定 性仅略有下降(图 S22 S 24 ), 表明这种原位聚合凝胶电 解质在高载量电极中也可发挥重要作用. 上述研究结果 表明, IPGE/ $\mathrm{H}_{2} \mathrm{SO}_{4}$ 器件在倍率性能、能量密度及循环稳 定性上都比 $\mathrm{PVA} / \mathrm{H}_{2} \mathrm{SO}_{4}$ 器件有较大提升, 在文献报道的 (准)固态非对称超级电容器中处于先进水平. 本研究提 供了一种新的用于构建准固态超级电容器的原位聚合 凝胶电解质.

\section{3 结论}

通过 N,S 共掺杂引入噟电容、改善浸润性, NSCNC 在 $1 \mathrm{~mol} \cdot \mathrm{L}^{-1} \mathrm{H}_{2} \mathrm{SO}_{4}$ 溶液、 $1 \mathrm{~A} \cdot \mathrm{g}^{-1}$ 下表现出 $337 \mathrm{~F} \cdot \mathrm{g}^{-1}$ 的 高比容量. 在相同的测试条件下, $\mathrm{WO}_{3} \bullet 0.6 \mathrm{H}_{2} \mathrm{O}$ 纳米棒在 $-0.55 \sim 0.3 \mathrm{~V} 、 5 \mathrm{~A} \cdot \mathrm{g}^{-1}$ 下表现出 $454 \mathrm{~F}^{\circ} \mathrm{g}^{-1}$ 的高比容量. 基于 $\mathrm{NSCNC}$ 和 $\mathrm{WO}_{3} \bullet 0.6 \mathrm{H}_{2} \mathrm{O}$ 互相匹配的比电容和电化 学窗口, 以 $\mathrm{NSCNC}$ 和 $\mathrm{WO}_{3} \cdot 0.6 \mathrm{H}_{2} \mathrm{O}$ 纳米棒作正负极组 装酸性介质非对称超级电容器, 通过原位聚合制备 $\mathrm{IPGE} / \mathrm{H}_{2} \mathrm{SO}_{4}$ 凝胶作为固态电解质, 建立了电解质-材料 之间的有效电荷传输界面, 降低了电荷转移电阻, 获得 了接近于液态器件、远优于传统 $\mathrm{PVA} / \mathrm{H}_{2} \mathrm{SO}_{4}$ 凝胶电解质 器件的电容性能. IPGE/ $\mathrm{H}_{2} \mathrm{SO}_{4}$ 电容器在 $1.5 \mathrm{~V}$ 电压下工 作, 在功率密度 $0.375 \mathrm{~kW} \cdot \mathrm{kg}^{-1}\left(0.5 \mathrm{~A} \cdot \mathrm{g}^{-1}\right)$ 时能量密度达 到 $22.31 \mathrm{Wh} \cdot \mathrm{kg}^{-1}$, 在功率密度 $7.5 \mathrm{~kW} \cdot \mathrm{kg}^{-1}\left(10 \mathrm{~A} \cdot \mathrm{g}^{-1}\right)$ 时 能量密度为 $8.55 \mathrm{Wh} \cdot \mathrm{kg}^{-1}$, 处于固态电容器的先进水平. $5 \mathrm{~A} \cdot \mathrm{g}^{-1}$ 下循环 4000 圈后比电容保持率为 $95.2 \%$, 循环稳 定性优于 $\mathrm{PVA} / \mathrm{H}_{2} \mathrm{SO}_{4}$ 器件. 本研究不仅展示了酸性介质 中 $\mathrm{NSCNC} / / \mathrm{WO}_{3} \bullet 0.6 \mathrm{H}_{2} \mathrm{O}$ 超级电容器的优异储能性能, 还提供了一种新的用于构建准固态超级电容器的原位 聚合凝胶电解质.

\section{4 实验部分}

\section{1 电极材料制备与表征}

采用本课题组发展的原位氧化镁模板法制备 $\mathrm{NSCNC}$, 吡啶和噻吩混合溶液 (体积比 $2: 1$ ) 为前驱 物 ${ }^{[12]}$. 按照文献报道方法制备 $\mathrm{WO}_{3} \bullet 0.6 \mathrm{H}_{2} \mathrm{O}$ 纳米棒 ${ }^{[15]}$. 称取 $1.76 \mathrm{~g} \mathrm{Na}_{2} \mathrm{WO}_{4} \cdot 2 \mathrm{H}_{2} \mathrm{O}$ 溶解于 $40 \mathrm{~mL}$ 蒸馏水中, 用 $\mathrm{HCl}$ 溶液 $\left(3 \mathrm{~mol} \cdot \mathrm{L}^{-1}\right)$ 调节 $\mathrm{pH} \approx 2$, 再加入 $1.41 \mathrm{~g}$
$\left(\mathrm{NH}_{4}\right)_{2} \mathrm{SO}_{4}$, 搅拌 $30 \mathrm{~min}$ 后将溶液转移到水热釜的聚四 氟乙烯罐中, 于 $180{ }^{\circ} \mathrm{C}$ 反应 $24 \mathrm{~h}$, 即可得到 $\mathrm{WO}_{3} \cdot 0.6 \mathrm{H}_{2} \mathrm{O}$ 纳米棒.

采用扫描电镜(SEM, Hitachi S4800)、透射电镜 (TEM, JEM-2100F)、X 射线光电子能谱(XPS, PHI 5000 VersaProbe)和 $\mathrm{X}$ 射线衍射(XRD, Bruker, D8 Advance $\mathrm{A} 25, \mathrm{Co}$ 靶) 表征样品形貌与组成. NSCNC 和 $\mathrm{WO}_{3} \cdot 0.6 \mathrm{H}_{2} \mathrm{O}$ 分别在 300 和 $100{ }^{\circ} \mathrm{C}$ 下脱气 $6 \mathrm{~h}$ 后在 Thermo Fisher Scientific Surfer Gas Adsorption Porosimeter 上进行氮气吸/脱附测试. 比表面积采用 Brunauer-Emmett-Teller (BET) 法计算得到. 在 $\mathrm{NETZSCH}$ 热分析仪上测试 $\mathrm{WO}_{3} \cdot 0.6 \mathrm{H}_{2} \mathrm{O}$ 中晶格水含量 (STA-499F3, 升温速率 $10{ }^{\circ} \mathrm{C} \cdot \mathrm{min}^{-1}$, 氩气作载气).

\section{2 电化学性能测试}

在 $\mathrm{H}$ 型电化学池中采用三电极系统测试电极材料 的电化学性能. 按质量比 $7: 1: 2$ 称取活性材料、聚偏 氟乙烯和导电碳(Super P), 加入适量乙醇作分散剂, 搅 拌 $12 \mathrm{~h}$ 至形成均匀浆料, 再涂覆于碳纸上用作工作电 极. 以 $\mathrm{Ag} / \mathrm{AgCl}$ 电极作参比、石墨棒作对电极、质子交 换膜作隔膜、 $1.0 \mathrm{~mol} \cdot \mathrm{L}^{-1} \mathrm{H}_{2} \mathrm{SO}_{4}$ 溶液作电解液, 在测试 过程中通入 $\mathrm{N}_{2}$ 保护.

以 $\mathrm{WO}_{3} \cdot 0.6 \mathrm{H}_{2} \mathrm{O}$ 作负极、 $\mathrm{NSCNC}$ 作正极、 $1.0 \mathrm{~mol} \cdot \mathrm{L}^{-1}$ $\mathrm{H}_{2} \mathrm{SO}_{4}$ 溶液作电解液, 在 $\mathrm{H}$ 型电化学池中组装非对称电 容器. 在 VMP3 电化学工作站上测试电化学性能 $\left(25{ }^{\circ} \mathrm{C}\right), \mathrm{CV}$ 和 $\mathrm{GCD}$ 测试的电压范围为 $0 \sim 1.5 \mathrm{~V}$. 器件 的电流密度以正负极活性物质总质量计算, 比电容根据 $\mathrm{GCD}$ 放电曲线结果计算得到:

$$
C_{\mathrm{wt}}=(I \Delta t) /[m(\Delta V-I R)]
$$

其中, $I$ 为放电电流, $\Delta t$ 为放电时间, $m$ 为活性材料总质 量, $\Delta V$ 为测试电压范围, $I R$ 为电压降.

\section{3 准固态非对称电容器的组装与测试}

IPGE/ $\mathrm{H}_{2} \mathrm{SO}_{4}$ 制备如下: 称取适量丙烯酰胺和 $N, N^{\prime}-$ 亚甲基双丙烯酰胺, 加入 $3 \mathrm{~mL} \mathrm{H}_{2} \mathrm{SO}_{4}$ 溶液 $\left(1.0 \mathrm{~mol} \cdot \mathrm{L}^{-1}\right)$ 中, 搅拌使之溶解. 依次加入适量 $\mathrm{H}_{2} \mathrm{O}_{2}$ 溶液 (100 $\left.\mathrm{mmol} \cdot \mathrm{L}^{-1}\right)$ 和抗坏血酸溶液 $\left(1 \mathrm{mg} \cdot \mathrm{mL}^{-1}\right)$ 作引发剂, 得到 前驱液. 将该前驱液置于 $50{ }^{\circ} \mathrm{C}$ 热处理 $30 \mathrm{~min}$, 即可原 位聚合形成 $\mathrm{IPGE} / \mathrm{H}_{2} \mathrm{SO}_{4}$ 凝胶电解质.

将工作电极放在 ITO 导电玻璃上, 滴加适量前驱 液, 使其与电极材料充分浸润, 以质子交换膜作隔膜, 以 ITO 玻璃为夹板和集流体组装成夹心型器件(图 S7), 加热使前驱液原位聚合形成凝胶电解质, 组装成准固态 非对称电容器. 在 VMP3 电化学工作站上测试电化学性 能 $\left(25^{\circ} \mathrm{C}\right)$, 测试电压范围为 $0 \sim 1.5 \mathrm{~V}$.

以含 $1.0 \mathrm{~mol} \cdot \mathrm{L}^{-1} \mathrm{H}_{2} \mathrm{SO}_{4}$ 溶液的 $\mathrm{PVA}$ 凝胶 ${ }^{[33]}$ 作为准 固态电解质组装成非对称电容器, 测试其性能作为对 照. 能量密度 $E$ 与功率密度 $P$ 的计算公式分别为:

$$
\begin{aligned}
& E=1 / 7.2 C V^{2} \\
& P=3.6 E / t
\end{aligned}
$$


其中, $C$ 为器件比电容, $V$ 为工作电压范围, $t$ 为放电时间.

\section{References}

[1] Raza, W.; Ali, F. Z; Raza, N.; Luo, Y. W.; Kim, K. H.; Yang, J. H.; Kumar, S.; Mehmood, A.; Kwon, E. E. Nano Energy 2018, 52, 441.

[2] Su, S. J.; Lai, Q. X.; Liang, Y. Y. Acta Chim. Sinica 2015, 73, 735. (苏善金, 来庆学, 梁彦瑜, 化学学报, 2015, 73, 735.)

[3] Muzaffar, A.; Ahamed, M. B.; Deshmukh, K.; Thirumalai, J. Renew. Sust. Energy Rev. 2019, 101, 123.

[4] Zhu, J.; Tang, S. C.; Wu, J.; Shi, X. L.; Zhu, B. G.; Meng, X. K. Adv. Energy Mater: 2017, 7, 1601234

[5] Couly, C.; Alhabeb, M.; Van Aken, K. L.; Kurra, N.; Gomes, L.; Navarro-Suarez, A. M.; Anasori, B.; Alshareef, H. N.; Gogotsi, Y. Adv. Electron. Mater. 2018, 4, 1700339.

[6] Zhao, J.; Andrew, F. B. Energy Storage Mater. 2021, 36, 31.

[7] Shao, Y.; El-Kady, M. F.; Sun, J.; Li, Y.; Zhang, Q.; Zhu, M.; Wang, H.; Dunn, B.; Kaner, R. B. Chem. Rev. 2018, 118, 9233.

[8] Wu, Q.; Yang, L.; Wang, X.; Hu, Z. Adv. Mater. 2020, 32, 1904177.

[9] Yang, L. J.; Shui, J. L.; Du, L.; Shao, Y. Y.; Liu, J.; Dai, L. M.; Hu, Z. Adv. Mater. 2019, 31, 1804799 .

[10] Wu, Q.; Yang, L.; Wang, X.; Hu, Z. Sci. China Chem. 2020, 63, 665

[11] Wu, Q.; Yang, L.; Wang, X.; Hu, Z. Acc. Chem. Res. 2017, 50, 435.

[12] Li, G.; Mao, K.; Liu, M.; Yan, M.; Zhao, J.; Zeng, Y.; Yang, L.; Wu, Q.; Wang, X.; Hu, Z. Adv. Mater. 2020, 32, 2004632.

[13] Zhao, J.; Lai, H.; Lyu, Z.; Jiang, Y.; Xie, K.; Wang, X.; Wu, Q.; Yang, L.; Jin, Z.; Ma, Y.; Liu, J.; Hu, Z. Adv. Mater. 2015, 27, 3541.

[14] Xie, K.; Qin, X.; Wang, X.; Wang, Y.; Tao, H.; Wu, Q.; Yang, L.; Hu, Z. Adv. Mater. 2012, 24, 347 .

[15] Jiang, H.; Hong, J. J.; Wu, X.; Surta, T. W.; Qi, Y.; Dong, S.; Li, Z.; Leonard, D. P.; Holoubek, J. J.; Wong, J. C.; Razink, J. J.; Zhang, X.; Ji, X. J. Am. Chem. Soc. 2018, 140, 11556.

[16] Anothumakkool, B.; Torris, A. A. T.; Veeliyath, S.; Vijayakumar, V.; Badiger, M. V.; Kurungot, S. ACS Appl. Mater. Interfaces 2016, 8, 1233.
[17] Lu, X. H.; Yu, M. H.; Wang, G. M.; Tong, Y. X.; Li, Y. Energy Environ. Sci. 2014, 7, 2160.

[18] Wang, F. X.; Wu, X. W.; Yuan, X. H.; Liu, Z. C.; Zhang, Y.; Fu, L. J.; Zhu, Y. S.; Zhou, Q. M.; Wu, Y. P.; Huang, W. Chem. Soc. Rev. 2017, 46, 6816

[19] Zhu, M.; Wu, J. X.; Wang, Y.; Song, M. M.; Long, L.; Siyal, S. H.; Yang, X. P.; Sui, G. J. Energy Chem. 2019, 37, 126.

[20] Anothumakkool, B.; Torris, A. A. T.; Bhange, S. N.; Unni, S. M.; Badiger, M. V.; Kurungot, S. ACS Appl. Mater. Interfaces 2013, 5, 13397.

[21] Niu, Y. B.; Yin, Y. X.; Wang, W. P.; Wang, P. F.; Guo, Y. G. CCS Chem. 2019, 1, 589 .

[22] Fan, H.; Wang, Y.; Gao, F.; Yang, L.; Liu, M.; Du, X.; Wang, P.; Yang, L.; Wu, Q.; Wang, X.; Hu, Z. J. Energy Chem. 2019, 34, 64.

[23] Augustyn, V.; Simon, P.; Dunn, B. Energy Environ. Sci. 2014, 7, 1597.

[24] Lu, S. Y.; Jin, M.; Zhang, Y.; Niu, Y. B.; Gao, J. C.; Li, C. M. $A d v$ Energy Mater. 2018, 8, 1702545.

[25] Luo, J.; Jang, H. D.; Huang, J. ACS Nano 2013, 7, 1464.

[26] Yan, J.; Fan, Z.; Sun, W.; Ning, G.; Wei, T.; Zhang, Q.; Zhang, R.; Zhi, L.; Wei, F. Adv. Funct. Mater. 2012, 22, 2632.

[27] Zhang, S. W.; Yin, B. S.; Wang, Z. B.; Peter, F. Chem. Eng. J. 2016, $306,193$.

[28] Subramani, K.; Sudhan, N.; Divya, R.; Sathish, M. RSC Adv. 2017, 7, 6648.

[29] Wang, C. Q.; Qiu, F. L.; Deng, H.; Zhang, X. Y.; He, P.; Zhou, H. S. Acta Chim. Sinica 2017, 75, 241. (王超强, 邱飞龙, 邓瀚, 张晓禹, 何平, 周豪慎, 化学学报, 2017, 75, 241.)

[30] Fan, H. L.; Niu, R. T.; Duan, J. Q.; Liu, W.; Shen, W. Z. ACS Appl. Mater. Interfaces 2016, $8,19475$.

[31] Liu, W. W.; Li, X.; Zhu, M. H.; He, X. J. Power Sources 2015, 282, 179.

[32] Lai, H.; Wu, Q.; Zhao, J.; Shang, L.; Li, H.; Che, R.; Lyu, Z.; Xiong, J.; Yang, L.; Wang, X. Energy Environ. Sci. 2016, 9, 2053.

[33] Gao, D.; Liu, R.; Yu, W.; Luo, Z.; Liu, C.; Fan, S. J. Phys. Chem. C 2019, 123, 5249 .

(Cheng, B.) 